\title{
ENVELHECIMENTO DE MISTURAS B5 E B20 EM TANQUES DE VEÍCULOS SUV
}

\author{
Ivna Oliveira da Cruz ${ }^{1}$, Maria Clara Kremer Faller ${ }^{1}$, Mauro lurk Rocha ${ }^{1}$, Tadeu \\ Massa $^{1}$ \\ ${ }^{1}$ CENPES, Petrobras - Petróleo Brasileiro S.A. \\ ivna-oliveira@petrobras.com.br \\ mcfaller@petrobras.com.br \\ miurk@petrobras.com.br \\ tcm@petrobras.com.br \\ antonio.vilella@petrobras.com.br \\ cmassa@petrobras.com.br
} Cavalcante Cordeiro de Melo ${ }^{1}$, Antonio Carlos Scardini Vilella, Carlos Vinicius Costa

\section{RESUMO}

O presente trabalho objetivou a avaliação, com foco em estabilidade, de misturas de biodiesel em óleo diesel nos percentuais de 5\% e 20\% v/v (B5 e B20) armazenadas nos tanques de veículos. Os testes foram conduzidos em veículos do tipo Sport Utility que permaneceram estacionados durante 9 meses. Durante 0 envelhecimento, foram retiradas amostras para análise de Estabilidade à Oxidação a $110^{\circ} \mathrm{C}$, Estabilidade à Estocagem, Número de Hidroperóxido, Número de Acidez Total, Teor de Zinco e de Cobre e também de Teor de Água. Para avaliar a dirigibilidade e o tempo de partida, foram realizadas duas partidas nos veículos, sendo a primeira com 4 meses de armazenamento e a outra, ao final do teste, após 9 meses. As amostras de B20, em comparação com B5, apresentaram resultados mais elevados de Insolúveis Totais, Índice de Acidez, Número de Hidroperóxidos e Teor de Água e resultados inferiores de Estabilidade de Oxidação, mas que não foram suficientes para impactar o tempo de partida dos veículos. Não foram constatados problemas de dirigibilidade com os veículos abastecidos com B5 e B20.

\section{INTRODUÇÃO}

No Brasil, a introdução do biodiesel na matriz energética brasileira ocorreu em 2005, pela Lei 11.097. Inicialmente, ficou estabelecido o uso autorizativo de $2 \%$ [1] em volume de biodiesel no óleo diesel comercializado, assim como a obrigatoriedade desta adição a todo o óleo diesel comercializado em território nacional a partir de $1^{\circ}$ de janeiro de 2008.

Uma das principais preocupações técnicas acerca do uso do biodiesel como combustível diz respeito à suscetibilidade à oxidação dessas substâncias quando em contato com o ar atmosférico. Essa característica está relacionada ao número de insaturações presentes nas cadeias carbônicas dos alquilésteres. Produtos de 
oxidação, como sedimentos e borras, podem se depositar no sistema de injeção dos veículos, afetando sua dirigibilidade. Sendo assim, com o avanço na tecnologia de motores do ciclo diesel e, pensando em um futuro aumento do teor de biodiesel a ser adicionado ao óleo diesel, essas preocupações técnicas relacionadas à estabilidade aumentam.

$\mathrm{Na}$ literatura [2-8], há diversos trabalhos sobre efeito da adição do biodiesel em emissões, desempenho e durabilidade dos veículos. No entanto, em relação ao efeito do biodiesel durante a estocagem em tanques de veículos, por um longo período, poucos trabalhos são encontrados.

Kono et al. [2] estudou o envelhecimento de misturas B20 em tanques de veículos. Em todos os veículos avaliados, não ocorreram problemas em até 7 meses de estocagem. Nos veículos equipados com tanques de metal, foram detectadas falhas no sistema de injeção e na partida do motor em diferentes períodos entre 8 e 18 meses. Os problemas foram relacionados à pressão insuficiente de combustível que ficou instável nos sistemas de injeção do tipo common rail.

Dentro desse contexto, o objetivo desse trabalho é avaliar o envelhecimento de misturas de óleo diesel com biodiesel, nos teores de 5 a $20 \%$ em volume, armazenadas em tanques de veículos do tipo Sport Utility (SUV).

\section{DESENVOLVIMENTO EXPERIMENTAL}

\subsection{Combustíveis}

A avaliação foi realizada com misturas B5 e B20, formuladas com óleo diesel S50 e biodiesel comercializados na época de sua condução (2012). Como premissa adotada, o biodiesel deveria apresentar estabilidade à oxidação limítrofe $(6$ horas). A tabela 1 apresenta os resultados de análise do biodiesel, utilizado nesse trabalho, de acordo com a especificação ANP nº 14 de 2012 [9].

Tabela I - Caracterização do biodiesel

\begin{tabular}{|l|c|c|c|c|}
\hline \multicolumn{1}{|c|}{ Proprie dades } & Unidade & Método & $\begin{array}{c}\text { Resolução ANP } \\
\mathbf{1 4 / 2 0 1 2}\end{array}$ & Biodiesel \\
\hline Aspecto & - & - & LII & LII \\
\hline Densidade a $20^{\circ} \mathrm{C}$ & $\mathrm{kg} / \mathrm{m}^{3}$ & ASTM D 4052 & $850-900$ & 881,8 \\
\hline Ponto de Fulgor & ${ }^{\circ} \mathrm{C}$ & ASTM D 93 & 100 & 128,8 \\
\hline Viscosidade a $40^{\circ} \mathrm{C}$ & $\mathrm{cSt}\left(\mathrm{mm}^{2} / \mathrm{s}\right)$ & ASTM D 445 & $3-6$ & 4,156 \\
\hline Microresíduo de Carbono & $\%$ massa & ASTM D 4530 & 0,05 & 0,00 \\
\hline Cinzas Sulfatadas & $\%$ massa & ASTM D 874 & 0,02 & $1 \mathrm{~A}$ \\
\hline Corrosividade ao Cobre & - & ASTM D 130 & 1 & 244 \\
\hline Teor de Água & $\mathrm{mg} / \mathrm{kg}$ & ASTM D6304 & 380 & 0,01 \\
\hline Teor de Glicerina Livre & $\% \mathrm{massa}$ & ASTM D 6584 & 0,02 & 0,20 \\
\hline Teor de Glicerina Total & $\% \mathrm{massa}$ & ASTM D 6584 & 0,25 & 0,60 \\
\hline Teor de Monoglicerídeo & $\%$ massa & ASTM D 6584 & 0,80 & 0,15 \\
\hline Teor de Diglicerídeo & $\%$ massa & ASTM D 6584 & 0,20 & 0,14 \\
\hline Teor de Triglicerídeo & $\%$ massa & ASTM D 6584 & 0,20 & 0,11 \\
\hline Teor de Metanol & $\%$ massa & CENPES & 0,20 & 3,86 \\
\hline Contaminação Total & mg/kg & EN 12662 & 24 & 0,25 \\
\hline Número de Acidez & mg KOH/g & ASTM D 664 & 0,50 & 6 \\
\hline Estabilidade a Oxidação a $110^{\circ} \mathrm{C}$ & EN 14112 & h & 6 & \\
\hline
\end{tabular}




\subsection{Veículos}

Foram utilizados quatro veículos na avaliação, do tipo Sport Utility (SUV) com sistema de injeção tipo common rail, ano 2011, baixa quilometragem de rodagem (inferior a $6000 \mathrm{~km}$ ) e que atendiam aos limites de emissão do Proconve L4. Dois veículos foram abastecidos com a mistura B5, identificados como B5-1 e B5-2, e mais dois veículos foram abastecidos com B20, identificados como B20-1 e B202.

\subsection{Procedimento do Teste de Envelhecimento}

No teste de envelhecimento, os veículos permaneceram estacionados durante 9 meses em uma área descoberta, exposta ao tempo, com incidência de sol, chuva e umidade do ambiente. Durante o período de estocagem, amostras de combustíveis foram coletadas para caracterização físico-química e os veículos também foram analisados quanto à dirigibilidade e tempo de partida. A etapa inicial da avaliação consistiu de um estresse térmico do combustível após abastecimento dos veículos, que rodaram no dinamômetro em $5^{\text {a }}$ marcha, velocidade constante e rotação de $2000 \mathrm{rpm}$ até que a temperatura do combustível atingisse $70^{\circ} \mathrm{C}$. Em seguida, os veículos foram levados para a área descoberta e permaneceram parados para início do teste de envelhecimento.

\subsection{Métodos Analíticos}

A avaliação físico-química dos combustíveis teve como foco a estabilidade. Durante os primeiros quatro meses de teste, a coleta de amostras foi quinzenal e, em seguida, passou a ser mensal até os 9 meses restantes. Os combustíveis coletados foram analisados quanto à estabilidade termo-oxidativa, formação de hidroperóxido, teor de metais e acidez. Em relação aos veículos, a avaliação da dirigibilidade e tempo de partida foi realizada duas vezes, uma após 4 meses e outra no final do teste.

\section{RESULTADOS E DISCUSSÕES}

Os resultados obtidos na avaliação de envelhecimento dos combustíveis nos tanques dos veículos B5-1, B5-2, B20-1 e B20-2 nos tanques dos veículos durante 9 meses são apresentados nas figuras 1 a 6 .

A figura 1 contempla os resultados de Insolúveis Totais, determinados pelo método acelerado de estabilidade à estocagem durante 16 horas a $90^{\circ} \mathrm{C}$ [10]. No eixo $x$, encontra-se o tempo em dias, onde o t125 (4 meses) representa a primeira partida dos veículos e o t260, representa a segunda partida já no final do teste. 


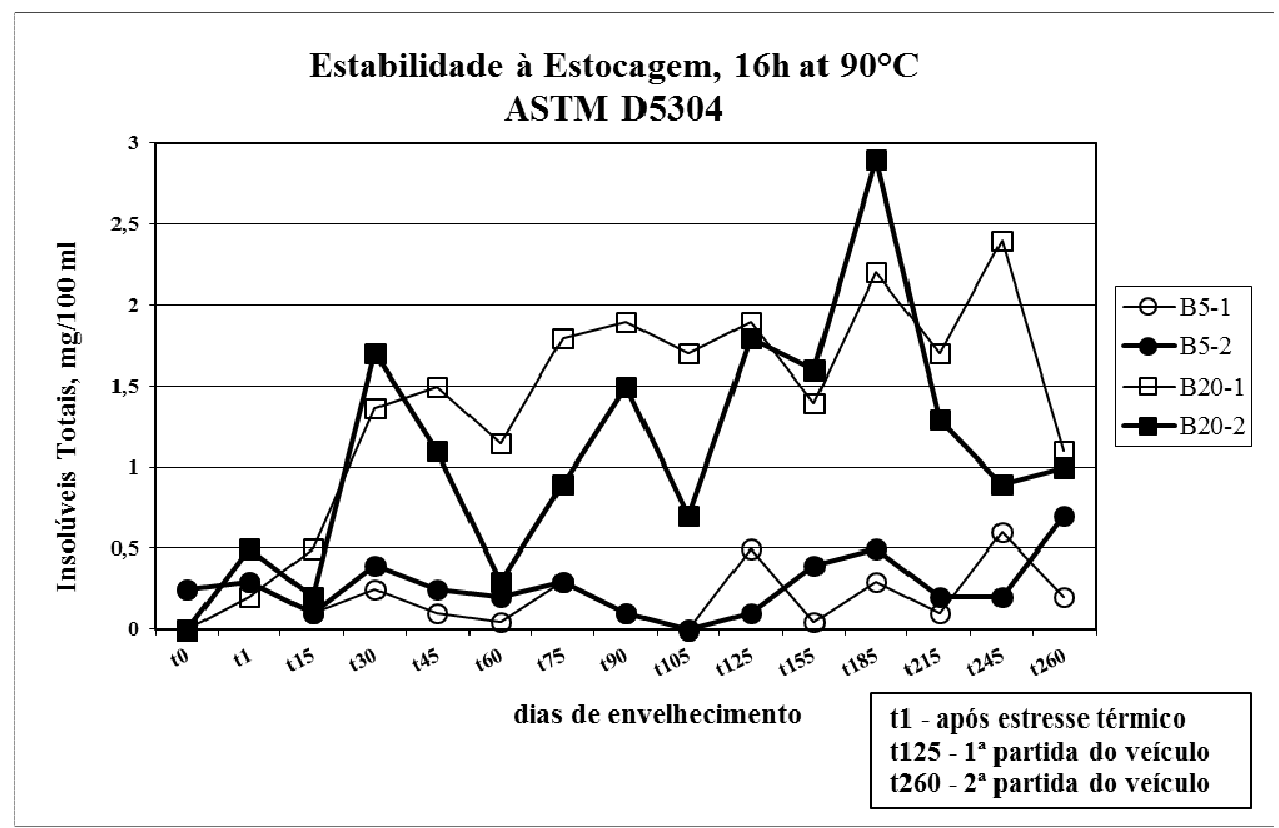

Figura 1 - Resultados de Insolúveis Totais durante envelhecimento

As amostras de B5 apresentaram níveis mais baixos de Insolúveis Totais, quando comparadas com B20. No entanto, as amostras de B20 continuaram atendendo a especificação ANP $\mathrm{n}^{\circ} 65$ de 2011 [11] de 2,5 mg/100ml máximo, considerando a elevada reprodutibilidade do método, na ordem de $1,4 \mathrm{mg} / 100 \mathrm{ml}$.

Os resultados de Período de Indução, determinado pelo método Estabilidade à Oxidação a $110^{\circ} \mathrm{C}$ [12], estão na figura 2 .

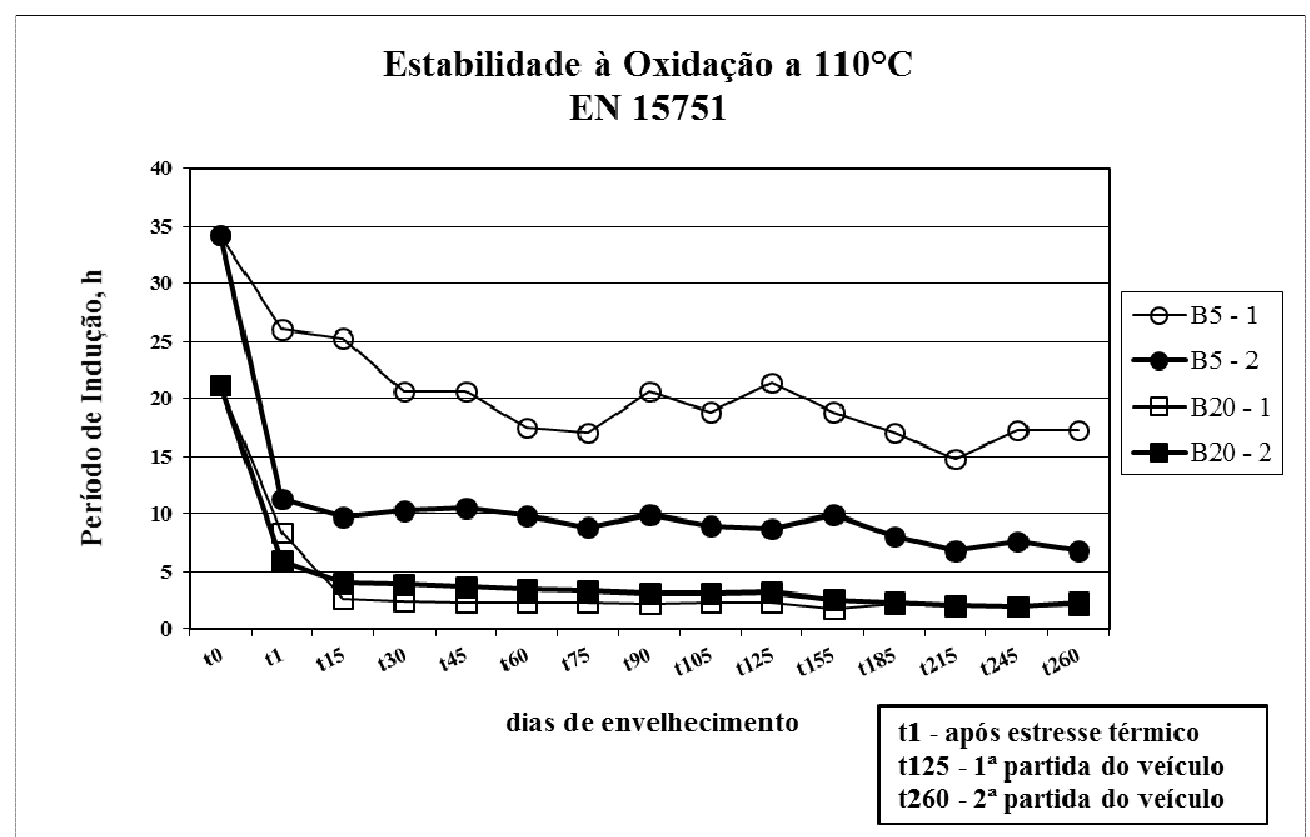

Figura 2 - Resultados de Período de Indução durante envelhecimento

Durante o envelhecimento, amostras de B5 apresentaram comportamento diferenciado. Enquanto a amostra B5-1 apresentou uma leve redução do período de indução após o estresse térmico do combustível, foi observado na amostra 
B5-2, um decréscimo significativo na estabilidade, assim como as amostras de B20. Porém, no caso de B20, as amostras B20-1 e B20-2, passaram a não atender ao limite de 20 horas de estabilidade à oxidação constante da especificação ANP n 2 de 2011 para misturas B6 a B20 [13].

A figura 3 apresenta os resultados da formação de hidroperóxido durante envelhecimento.

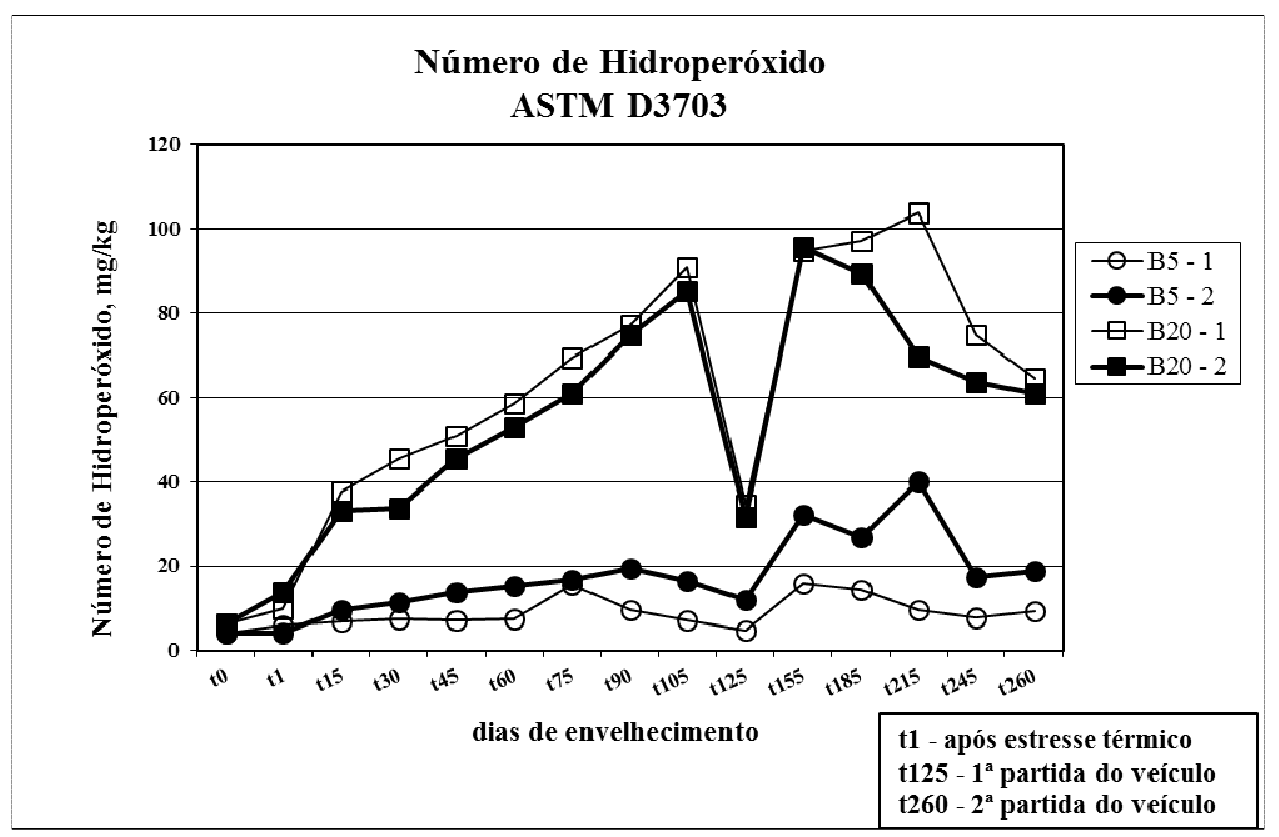

Figura 3 - Resultados de Hidroperóxido durante envelhecimento

As formulações avaliadas apresentaram um aumento do número de hidroperóxido com o tempo em função da ocorrência das reações de oxidação, principalmente nos primeiros 4 meses do envelhecimento (t125), sendo mais significativo nas amostras B20-1 e B20-2. Após a primeira partida dos veículos, foi observada uma tendência de queda na formação de hidroperóxido nas misturas B20, que pode ser atribuído ao esgotamento do processo oxidativo.

A acidez das amostras coletadas também foi analisada e os resultados estão dispostos na figura 4 . 


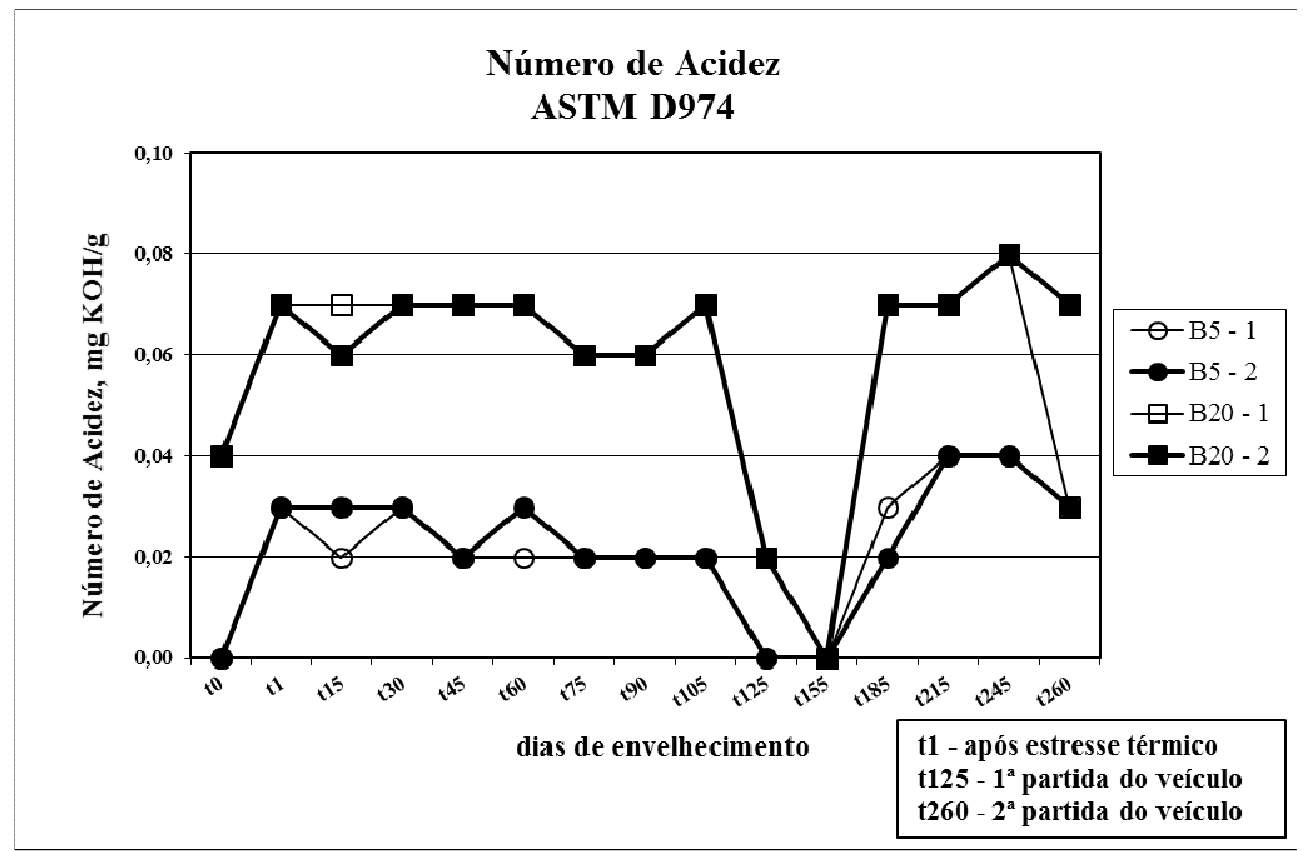

Figura 4 - Resultados de Número de Acidez durante envelhecimento

Não foram observadas alterações significativas do Número de Acidez das formulações B5 e B20 durante os 9 meses de envelhecimento. Como já era esperado, as amostras B20 apresentaram acidez mais elevada que as amostras de B5.

Os resultados das análises de metais (Teor de Cobre e Teor de Zinco) estão apresentados nas figuras 5 e 6 .

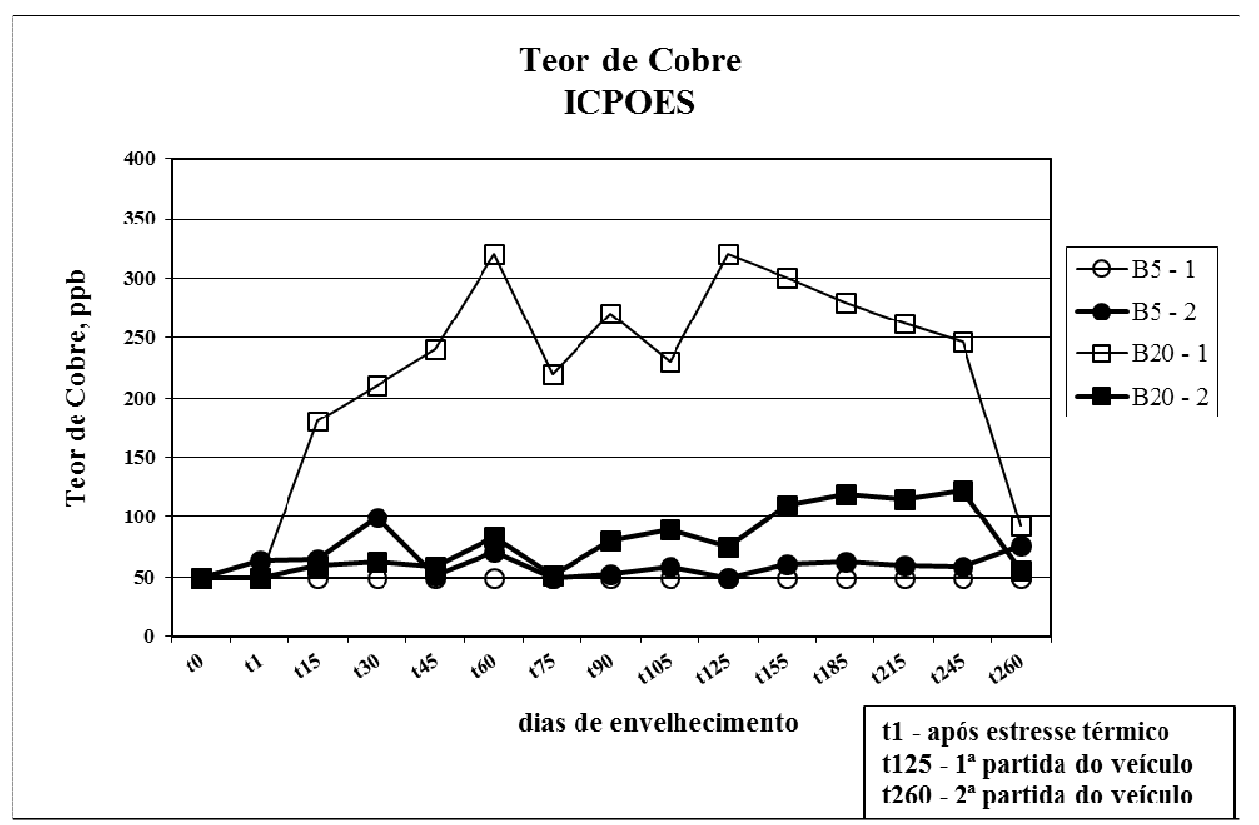

Figura 5 - Resultados de Teor de Cobre durante envelhecimento

As formulações de B5 apresentaram baixos teores de cobre, sempre inferiores a $100 \mathrm{ppb}$. Por outro lado, amostras de B20 apresentaram comportamento diferenciado nos veículos avaliados. Enquanto B20-2 apresentou teores de cobre 
inferiores a $150 \mathrm{ppb}$, a amostra do outro veículo, B20-1, apresentou teores bem mais elevados durante o envelhecimento.

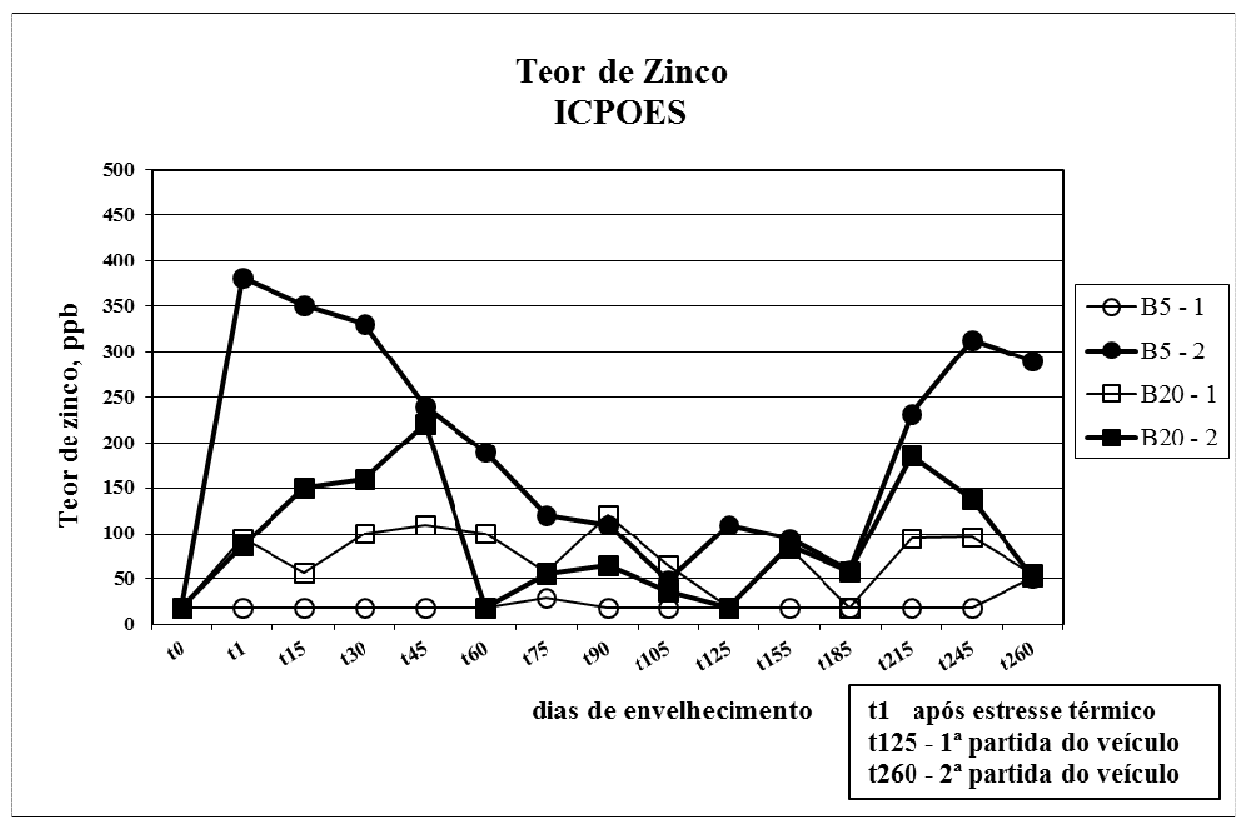

Figura 6 - Resultados de Teor de Zinco durante o envelhecimento

Assim como ocorreu com o cobre, os teores de zinco nas amostras avaliadas foram diferentes. Enquanto as misturas B20 apresentaram teores de zinco baixos, uma amostra de B5 mostrou um aumento significativo desse metal durante o envelhecimento. Vale ressaltar que teores de metais, tais como zinco e cobre, mesmo na faixa de 20 ppb, podem catalisar reações de oxidação, levando a formação de insolúveis que tendem a provocar entupimento do sistema de injeção e de filtro.

Para finalizar, na tabela II, são apresentados os resultados de tempo de partida, em segundos, dos 4 veículos SUVs usados no trabalho.

Tabela II - Resultados de tempo de partida

\begin{tabular}{|c|c|c|}
\hline \multirow{2}{*}{ Veículo } & \multicolumn{2}{|c|}{ Tempo de partida (s) } \\
\cline { 2 - 3 } & $\begin{array}{c}\text { Primeiro teste } \\
\text { (t125) }\end{array}$ & $\begin{array}{c}\text { Segundo teste } \\
(\mathbf{t 2 6 0})\end{array}$ \\
\hline $\mathbf{B 5 - 1}$ & 0,777 & 1,156 \\
\hline $\mathbf{B 5 - 2}$ & 1,033 & 2,603 \\
\hline $\mathbf{B 2 0 - 1}$ & 0,774 & 1,012 \\
\hline $\mathbf{B 2 0 - 2}$ & 0,773 & 1,160 \\
\hline
\end{tabular}

Não foram observados problemas de dirigibilidade durante as duas partidas realizadas (t125 e t260). Por outro lado, em relação ao tempo de partida, o 
veículo abastecido com B5-2 apresentou um atraso mais significativo em comparação aos demais, porém isso não comprometeu sua dirigibilidade.

\section{CONCLUSÕES}

Os resultados da avaliação físico-química mostraram que a deterioração das misturas de 5\% e 20\%, em volume, de biodiesel em óleo diesel, B5 e B20, armazenadas em tanques de veículos parados por um período de 9 meses não foi suficiente para impactar no tempo de partida e na dirigibilidade de veículos.

As formulações de B5 (B5-1 e B5-2) e de B20 (B20-1 e B20-2) avaliadas atenderam às especificações da ANP e apresentaram valores baixos de Número de Hidroperóxido, Teor de Água e Índice de Acidez, assim como estabilidades à estocagem e à oxidação adequadas, no caso de B5, e Período de Indução limítrofe para B20.

Durante o envelhecimento, as amostras B20-1 e B20-2, em comparação com B5-1 e B5-2, apresentaram resultados mais elevados de Insolúveis Totais, Índice de Acidez, Número de Hidroperóxidos e Teor de Água e resultados inferiores de Estabilidade à Oxidação, não atendendo ao limite mínimo de 20 horas da especificação ANP para uso experimental de B6 a B20.

Por fim, vale ressaltar que tais resultados são pontuais e obtidos em condições adequadas de manuseio e estocagem dos combustíveis. Sendo assim, novas avaliações de misturas contendo $20 \%$ de biodiesel em óleo diesel são necessárias considerando as variabilidades presentes em aplicações reais de campo.

\section{REFERÊNCIAS BIBLIOGRÁFICAS}

[1] Agência Nacional do Petróleo, Gás Natural e Biocombustíveis. RESOLUÇÃO ANP № 42 de 24.11.2004.

[2] Kono, N., Yamamori K., Furukawa, T., Noorman, M., "FAME Blended Diesel Fuel Impacts on Engine/Vehicle Systems", SAE Technical Paper 2011-01-1944, 2011.

[3] Richard Fraer, Han Dinh, Kenneth Proc, Robert L.McCormick, Kevin Chandler, BuchholzBruce Buchholz, "Operating Experience and Teardown Analysis for Engines Operated on Biodiesel Blends (B20)", SAE Technical Paper 2005-01-3641, 2005.

[4] Kenneth Proc, Robb Barnitt, R. Robert Hayes,Matthew Ratcliff, Robert L. McCormick, Lou Ha,Howard L. Fang, "100,000-Mile Evaluation of Transit Buses Operated on Biodiesel Blends (B20)", SAE Technical Paper 2006-01-3253, 2006. 
[5] Robert Quigley, Robert H. Barbour, "Biodiesel Quality Improvement with Additive Treatment", SAE Technical Paper 2004-01-1859, 2004.

[6] Itsuki Miyata, Yasunori Takei, Kazushi Tsurutani, Masanori Okada, "Effects of BioFuels on Vehicle Performance: Degradation Mechanism Analysis of Bio-Fuels", SAE Technical Paper 2004-01-3031, 2004.

[7] Barbara Terry, Robert L. McCormick, Mani Natarajan, "Impact of Biodiesel Blends on Fuel System Component Durability", SAE Technical Paper 2006-01-3279, 2006.

[9] Agência Nacional do Petróleo, Gás Natural Biocombustíveis. RESOLUÇÃO ANP № 14 de 11.05.2012.

[10] AMERICAN SOCIETY FOR TESTING AND MATERIALS D 5304 - 11: Standard Test Method for Assessing Middle Distillate Fuel Storage Stability by Oxygen Overpressure: ASTM International, 2012.

[11] Agência Nacional do Petróleo, Gás Natural e Biocombustíveis. RESOLUÇÃO ANP № 65 de 9.12.2011.

[12] EN 15751 Automotive fuels - Fatty acid methyl esters (FAME) and blends with diesel fuel - Determination of oxidation stability by accelerated oxidation method. CEN, 2009.

[13] Agência Nacional do Petróleo, Gás Natural e Biocombustíveis. RESOLUÇÃO ANP № 2 de 12.01.2011. 
